\title{
SEROPREVALENCIA DE LEPTOSPIROSIS BOVINA EN EL CHACO CENTRAL, DEPARTAMENTO DE BOQUERÓN, PARAGUAY
}

\author{
BOVINE LEPTOSPIROSIS SEROPREVALENCE IN CENTRAL CHACO, \\ BOQUERÓN DEPARTMENT, PARAGUAY
}

\author{
Szwako $A^{1}$, Acuña $L^{2}$, Rolón $C^{1}$, Glatzle $F^{3}$, Lemkemeyer $C^{3}$, Unger $\mathbf{N}^{3}$, Wiebe $\mathbf{J}^{3}$ \\ ${ }^{1}$ Cátedras de "Patología y Clínica de los Rumiantes" - Facultad de Ciencias Veterinarias - Universidad Nacional de Asunción - San \\ Lorenzo - Paraguay \\ ${ }^{2}$ Departamento de Salud Pública - Facultad de Ciencias Veterinarias - Universidad Nacional de Asunción - San Lorenzo - Paraguay \\ ${ }^{3}$ Tesistas de Grado para optar al título de Doctor en Ciencias Veterinarias - Facultad de Ciencias Veterinarias - Universidad Nacional \\ de Asunción - San Lorenzo - Paraguay
}

RESUMEN. En las Colonias Fernheim, Neuland y Menno del Chaco Central, departamento de Boquerón, Paraguay, se seleccionaron 31 establecimientos representativos de la población, tomándose al azar 415 muestras sanguíneas, con el fin de determinar la seroprevalencia de leptospirosis. En la muestra se incluyeron animales de ambos sexos, mayores a dos años. No se muestrearon vacas que se encontraban a 30 días del pre o postparto, tampoco aquellos bovinos vacunados hasta 3 meses previos a la toma de la muestra. Se procesaron las muestras por la prueba de seroaglutinación microscópica estandarizada incluyendo 11 serovares de Leptospira interrogans, considerándose positivos los títulos a partir de 1:100. Resultaron positivas 190 muestras (45,78\%) para uno o más serovares, de los cuales los más frecuentes fueron wolffi $(77,41 \%)$ y hardjo $(22,59 \%)$; no habiendo reacción de micro aglutinación para los serovares: icterohaemorrhagiae, borincana, canicola, ballum, pyrogenes, pomona, bratislava, tarassovi y moskva. De los animales positivos se presentó reacción simultánea a los serovares wolffi y hardjo en 49 muestras $(25,8 \%)$. Con la investigación se concluye que la leptospirosis se encuentra difundida en el ganado bovino del Chaco Central, recomendando la implementación de medidas de prevención de la enfermedad.

Palabras clave: seroprevalencia, leptospirosis, ganado bovino, Chaco Central, Paraguay.

ABSTRACT. At Fernheim, Neuland and Menno Colonies, Central Chaco, Boqueron department, Paraguay, 31 cattle ranches were selected, representative of the population, 415 blood samples obtained randomly, in order to determine leptospirosis seroprevalence. Both sexes and older than two years animals were included. No cows at 30 days from pre- or postpartum were sampled, neither those vaccinated cattle up to 3 months prior to sampling. Blood serum samples were processed by the standarized microscopic seroagglutination test including 11 serovars of Leptospira interrogans, considering positive from 1:100 titers. 190 samples were positive (45.78\%) to one or more serovars, of which the most frequent were wolffi $(77.41 \%)$ and hardjo (22.59\%); not having micro agglutination reaction to the serovars: icterohaemorrhagiae, borincana, canicola, ballum, pyrogenes, pomona, bratislava, tarassovi and moskva. Simultaneous positives results showed reaction to wolffi and hardjo serovars in 49 samples (25.8\%). This investigation concluded that leptospirosis is widespread in Central Chaco cattle, recommending implementation of disease prevention measures.

Keywords: seroprevalence, leptospirosis, bovine, Central Chaco, Paraguay.

doi: http://dx.doi.org/10.18004/compend.cienc.vet.2015.05.01.26-30

Dirección para correspondencia: Prof. Dr. Alexander Szwako - Departamento de Investigación Científica y Tecnológica Facultad de Ciencias Veterinarias - Universidad Nacional de Asunción - Casilla de Correo № 1061 - Ruta Mcal. Estigarribia Km 10,5 - Campus Universitario - San Lorenzo - Paraguay

E-Mail: aszwako@vet.una.py

Recibido: 05 de marzo de 2015 / Aceptado: 15 de junio de 2015 


\section{INTRODUCCIÓN}

La leptospirosis es una enfermedad bacteriana zoonótica distribuida mundialmente y de epidemiología compleja, producida por la infección con espiroquetas del género Leptospira que, clásicamente, comprende dos especies: $L$. interrogans y L. biflexa, siendo la primera patógena y la segunda, saprófita. L. interrogans incluye alrededor de 23 serogrupos y 218 serovares. La clasificación en serovares se basa en técnicas de microaglutinación. Los serovares antigénicamente relacionados se agrupan en serogrupos. Afecta a la mayoría de los mamíferos domésticos. También se ha documentado en reptiles, aves, anfibios y artrópodos (1-4).

La leptospirosis en humanos fue descrita en 1886, siendo denominada enfermedad de Weil $(4,5)$; el agente etiológico, fue aislado en 1915, por Inada e Ido, en Japón. Noguchi, en 1917, estableció finalmente el género Leptospira, aislando el microorganismo de un ratón, por primera vez $(5,6)$. La infección humana se relaciona, principalmente, con riesgo laboral y recreacional, pudiendo infectarse el hombre por contacto directo con el reservorio animal o, más frecuentemente, a través de agua o terrenos húmedos contaminados (agua estancada, estanques, arrozales, etc) (4). Es considerada una enfermedad reemergente, de distribución mundial, con comportamiento endémico y brotes en varios continentes (7).

En el ganado bovino puede cursar con diferentes cuadros clínicos, pudiendo presentarse como un cuadro agudo/hiperagudo con fiebre, hemoglobinuria y meningitis hasta incluso la muerte, a su vez en el cuadro crónico la única sintomatología aparente es el fallo reproductivo (1). La enfermedad causa pérdidas económicas, a consecuencia de abortos, infertilidad, terneros que nacen débiles y mueren en los primeros días de vida y por disminución de la producción láctea (2). En caso de mastitis, la leche se caracteriza por ser espesa, descolorida o amarilla y manchada de sangre pero sin inflamación mamaria $(3,8)$.

La prueba diagnóstica que se utiliza comúnmente es la prueba de seroaglutinación microscópica estandarizada (MAT), la cual detecta inmunoglobulinas $\mathrm{M}$ (IgM) que corresponden únicamente a estados agudos de la enfermedad. La
MAT sigue siendo la prueba estándar para el diagnóstico de leptospirosis porque posee elevada sensibilidad en la detección de la IgM frente a serovares específicos $(3,7)$; no obstante entre sus principales desventajas se mencionan la no diferenciación entre anticuerpos vacunales y de infección, además de utilizar como antígeno leptospiras vivas, siendo engorroso el mantenimiento de las cepas y un riesgo potencial para el personal del laboratorio (1).

El objetivo de este estudio fue determinar la seroprevalencia de serovares de L. interrogans que afectan al ganado bovino en la Colonia Fernheim, Neuland y Menno del Chaco Central, Departamento de Boquerón, Paraguay.

\section{MATERIALES Y MÉTODOS}

Cuatrocientos quince (415) bovinos, machos y hembras, mayores a 2 años de edad, de aptitud lechera y carnicera fueron muestreados de 31 establecimientos (codificado 1 a 31) para la realización de este estudio, contando previamente con el consentimiento de los productores. Los animales fueron de las siguientes razas: Holando (203), Brahman (81), Mestiza cebú (73), Gelbvieh (23), Nelore (10), Fleckvieh (9), Jersey (6), Braford (5), Santa Gertrudis (3) y Pardo suizo (2). Algunos de estos animales presentaron fallas reproductivas. Fueron excluidos del muestreo las vacas que se encontraban 30 días pre y postparto o post aborto, y animales en los cuales no transcurrieron 3 meses de intervalo de tiempo entre la última vacunación y la toma de muestra. De los 31 establecimientos muestreados, 15 fueron de sistema de producción de leche semi-intensivo (tambos) y 16 de cría/recría de ganado de corte en forma extensiva, localizados en las Colonias Fernheim, Neuland y Menno (Coordenadas geográficas: $22^{\circ} 21^{\prime} 8.14^{\prime \prime} \mathrm{S}$ $60^{\circ} 2^{\prime} 5.85^{\prime \prime} 0 ; 22^{\circ} 38^{\prime} 46.88^{\prime \prime S}$ 607'51.22"0; $22^{\circ} 23^{\prime} 19.65^{\prime \prime S} 59^{\circ} 50^{\prime} 10.98^{\prime \prime} 0$, respectivamente), todas de la zona del Chaco Central, Departamento de Boquerón, Paraguay.

Una vez realizada la sujeción segura del animal en la casilla de operaciones, se registró la identificación del mismo y se determinó la edad. Se limpió la región coccígea con agua y/o papel desechable y se realizó la antisepsia con algodón embebido en alcohol etílico de $95^{\circ}$. A continuación se tomó con aguja descartable № 18 y jeringa de $10 \mathrm{ml}$ 
este mismo volumen de sangre de cada animal, mediante punción de la vena/arteria coccígea media a nivel del tercer espacio intercoccígeo. Luego se depositó la sangre en tubos estériles debidamente identificados y se dejó reposar a temperatura ambiente durante 30 - $45 \mathrm{~min}$ en una posición inclinada $\left(45^{\circ}\right)$ para facilitar la coagulación. El suero fue separado del coágulo para ser almacenado a una temperatura de $-18^{\circ} \mathrm{C}$ hasta su procesamiento laboratorial.

Las muestras se remitieron al Laboratorio de Patología y Control de Alimentos - División Bacteriología (Leptospirosis) del Servicio Nacional de Salud y Calidad Animal (SENACSA) para su procesamiento por medio del método de MAT (prueba de seroaglutinación microscópica estandarizada) (9).

Los antígenos utilizados corresponden a las cepas tipo que identifican a cada una de las distintas serovariedades importantes de la clasificación actual. Utilizándose los siguientes antígenos: icterohaemorrhagiae, borincana, canicola, ballum, hardjo, pyrogenes, pomona, bratislava, tarassovi, wolffi y moskva; considerándose positivos los títulos a partir de 1:100.

Los resultados fueron analizados usando el método de estadística descriptiva. Fueron evaluadas las frecuencias absoluta y relativa de los animales positivos en los establecimientos estudiados y de la titulación serológica de aglutinación por $L$. interrogans.

\section{RESULTADOS Y DISCUSIÓN}

Se determinó una seroprevalencia general del 45,78\% (190/415) para uno o más serovares encontrándose los títulos a partir de 1:200. Animales seropositivos fueron detectados en 27 de los 31 establecimientos muestreados (87\%)(Tabla 1), y sólo dos de los serotipos de Leptospira interrogans estuvieron presentes (wolffi y hardjo). La prevalencia es relevante considerando las condiciones climáticas, como el calor, las lluvias de verano, la presencia de tajamares y el suelo bajo en algunas zonas, factores que favorecen la presencia y supervivencia de las Leptospiras, además de observarse la presencia de posibles reservorios como perros, roedores y animales silvestres.
Tabla 1. Distribución de número de animales muestreados, positivos y porcentaje de positivos según el establecimiento de procedencia.

\begin{tabular}{|c|c|c|c|}
\hline $\begin{array}{c}\text { Establecimiento } \\
\text { № }\end{array}$ & $\begin{array}{c}\text { Número de } \\
\text { animales } \\
\text { muestreados }\end{array}$ & $\begin{array}{l}\text { Número de } \\
\text { animales } \\
\text { Positivos* }\end{array}$ & $\begin{array}{c}\text { Porcentaje } \\
\text { (\%) }\end{array}$ \\
\hline 1 & 19 & 10 & 52,63 \\
\hline 2 & 15 & 5 & 33,33 \\
\hline 3 & 17 & 3 & 17,65 \\
\hline 4 & 15 & 0 & 0,00 \\
\hline 5 & 20 & 8 & 40,00 \\
\hline 6 & 17 & 8 & 47,05 \\
\hline 7 & 12 & 0 & 0,00 \\
\hline 8 & 11 & 4 & 36,36 \\
\hline 9 & 11 & 0 & 0,00 \\
\hline 10 & 6 & 4 & 66,66 \\
\hline 11 & 10 & 5 & 50,00 \\
\hline 12 & 10 & 3 & 30,00 \\
\hline 13 & 11 & 6 & 54,54 \\
\hline 14 & 11 & 8 & 72,72 \\
\hline 15 & 12 & 8 & 66,66 \\
\hline 16 & 6 & 4 & 66,66 \\
\hline 17 & 17 & 9 & 52,94 \\
\hline 18 & 25 & 10 & 40,00 \\
\hline 19 & 24 & 15 & 62,50 \\
\hline 20 & 19 & 12 & 63,15 \\
\hline 21 & 27 & 25 & 92,59 \\
\hline 22 & 10 & 2 & 20,00 \\
\hline 23 & 10 & 3 & 30,00 \\
\hline 24 & 10 & 6 & 60,00 \\
\hline 25 & 10 & 4 & 40,00 \\
\hline 26 & 10 & 9 & 90,00 \\
\hline 27 & 10 & 6 & 60,00 \\
\hline 28 & 10 & 7 & 70,00 \\
\hline 29 & 10 & 4 & 40,00 \\
\hline 30 & 10 & 2 & 20,00 \\
\hline \multicolumn{4}{|l|}{31} \\
\hline Total & 415 & 190 & $45,78^{* *}$ \\
\hline
\end{tabular}

En este estudio, se detectó presencia de anticuerpos a dos serovares: wolffi $(77,41 \%)$ y hardjo (22,59\%) como se observa en la Tabla 2, la cual demuestra una mayor frecuencia de los títulos de aglutinación en las diluciones 1:400 (122) y 1:800 (109). De los animales positivos se presentó reacción simultánea a los serovares wolffi y hardjo en 49 muestras $(25,8 \%)$. No se presentó reacción de micro a glutinación para los serovares: icterohaemorrhagiae, borincana, canicola, ballum, pyrogenes, pomona, bratislava, tarassovi y moskva.

Los resultados de prevalencia obtenidos en el presente estudio se aproximan a los encontrados por 
Tabla 2. Distribución de reacciones positivas por títulos de seroaglutinación para serovares de Leptospira interrogans, wolffi y hardjo.

\begin{tabular}{|c|c|c|c|c|c|c|}
\hline \multirow{2}{*}{ Serovar } & \multicolumn{4}{|c|}{ Titulación } & \multirow{2}{*}{ Total } & \multirow{2}{*}{$\begin{array}{c}\text { Porcentaje } \\
(\%)\end{array}$} \\
\hline & $1: 200$ & $1: 400$ & $1: 800$ & 1:1600 & & \\
\hline wolffi* & 1 & 98 & 81 & 5 & 185 & 77,41 \\
\hline hardjo* & & 24 & 28 & 2 & 54 & 22,59 \\
\hline Total & 1 & 122 & 109 & 7 & 239 & 100,00 \\
\hline
\end{tabular}

Oliveira et al (2001), quienes en el Distrito municipal de Garanhuns, Estado de Pernambuco - Brasil, encontraron una seroprevalencia del 47,63\% (10). Por su parte, Luna et al (2005) en la República de México, específicamente en la región de trópico seco determinaron una prevalencia de 45,9\% (11).

En otros estudios realizados en Brasil y México; Soares et al (2000) encontraron una seroprevalencia de $81,90 \%$ de animales reaccionantes, específicamente ganado lechero, de la microrregión de Goiania (5), Pimienta et al (2014) encontraron $61 \%$ de prevalencia en el Estado de Paraíba (12), y Moles et al (2002) determinaron un $73 \%$ de prevalencia en el altiplano central de la república Mexicana (13), valores por encima del encontrado en este estudio.

Nicolino et al (2014) encontraron en la microrregión de Sete Lagoas, Minas Gerais, Brasil una seroprevalencia de 20,7\% (14). Moles et al (2002) determinaron 31,1\% de prevalencia (15), así como Luna et al (2005) en la región árida y semiárida obtuvieron un 37,8\% (11), ambos en México. En Colombia, Zuluaga et al (2009) determinaron 16,4\% de animales positivos a leptospirosis (8), Ochoa (2000) en el Departamento de Antioquia, encontraron 10,3\% de las vacas en producción (7) y Motta et al (2014), por su parte hallaron 28\% de animales positivos en el Departamento de Caquetá (16). No obstante los valores mencionados resultaron ser inferiores a los encontrados en el Chaco Central paraguayo. La prevalencia de reacción serológica positiva a Leptospirosis varía considerablemente entre países, regiones, áreas e incluso establecimientos, este comportamiento epizootiológico de la patología podría deberse a la presentación de diferentes factores de riesgo en cada lugar.

En otro estudio realizado fuera del continente americano, específicamente en la Región Marmara, Turquía, Kocabiyik y Cetin (2004) identificaron una prevalencia de 3,4\%, cifra mucho menor a las mencionadas en estudios publicados en la región sudamericana y en este estudio (17).

En el presente estudio se han identificado dos serovares de leptospira, wolffi y hardjo, así como la combinación de ambos serovares en 49 muestras (25,8\%). Soares et al (2000) en Goiania, Brasil identificaron los serovares wolffi $(36,10 \%)$, icterohaemorrhagiae $(20,50 \%)$, hardjo $(5,20 \%)$ y tarassovi (4,90\%) (5); y Oliveira et al (2001) determinaron la siguiente distribución de serovariedades: hardjo $(21,98 \%)$ bratislava $(15,73 \%)$ castellonis $(11,64 \%)$ tarassovi $(10,56 \%)$ pyrogenes $(1,72 \%)$ icterohaemorrhagiae $(1,08 \%)$, pomona $(0,86 \%)$, grippotyphosa $(0,86 \%)$, djasiman $(0,43 \%)$, canicola $(0,21 \%)$ y copenhageni $(0,21 \%)$ (10), en el Distrito municipal de Garanhuns, Estado de Pernambuco, Brasil, coincidiendo parcialmente en ambos casos con el presente estudio. Por su parte, Moles et al (2002) y Luna et al (2005) en México, también han identificado serovares hardjo y wolffi, como los principales, aunque además han aislado el serovar tarassovi $(11,13)$.

Nicolino et al (2014) en Minas Gerais y Pimienta et al (2014) en el Estado de Paraíba, Brasil, así como también Zuluaga et al (2009) en Colombia han identificado solamente hardjo, en estos casos coincidiendo con uno de los serovares identificados $(8,12,14)$.

\section{CONCLUSIÓN}

La leptospirosis se encuentra presente en el ganado bovino del Chaco Central paraguayo, con una prevalencia considerable $(45,78 \%)$, teniendo en cuenta que las condiciones medioambientales (temperatura y humedad) existentes fueron favorables a la sobrevivencia del microorganismo y permitieron su transmisión. Los serovares más frecuentes fueron wolffi $(77,41 \%)$ y hardjo $(22,59 \%)$ con reacción simultánea a ambos serovares en el 


\section{$25,8 \%$ de las muestras.}

Los resultados obtenidos justifican la implementación de medidas de prevención de la enfermedad y sugieren el desarrollo de investigaciones que determinen el impacto económico y sanitario de la enfermedad en ésta y otras regiones del país.

\section{AGRADECIMIENTOS}

A las autoridades del Servicio Nacional de Salud y Calidad Animal (SENACSA), al equipo profesional del Laboratorio de Patología y Control de Alimentos - División Bacteriología (Leptospirosis) y a los productores, por permitir la realización del presente estudio.

\section{BIBLIOGRAFÍA}

1- Alonso-Andicoberry C, García-Peña FJ, Ortega-Mora LM Epidemiología, diagnóstico y control de la leptospirosis bovina (Revisión). Invest. Agr. Prod. Sanid. Anim. 2001; (16): 205-225.

2- Draghi MG, Brihuega B, Benítez D, Sala JM, Biotti GM, Pereyra M, Homse A, Guariniello L. Brote de leptospirosis en terneros en recría en la provincia de Corrientes, Argentina. Rev Argent Microbiol. 2011; (43): 42-44.

3- Aricapa HJ, Pérez JE, Cabrera IC, Rivera K. Valoración de la respuesta de anticuerpos tipo IgM e IgG frente a leptospira en bovinos. Biosalud. 2008 enero - diciembre; (7): 29-39.

4- Zunino E, Pizarro R. Leptospirosis: puesta al día. Rev Chil Infect. [Revista on- line]. 2007; 24 (3): 220-226.

5- Soares R, Troncoso N, Acyprestes C, Souza L, Queiroz H, Rodrigues L, Gottschalk S, Caetano R. Prevalência e aspectos epidemiológicos da leptospirose bovina em rebanho leiteiro na microrregião de Goiânia - GO. Cienc. Rural [Revista on-line]. 2000; 30 (5): 857-862.

6- Steele JH, Mildred MPH, Galton M. et al. Leptospirosis as a world problem. Veterinary Medicine. 1957; 3 (11): 517-527.

7- Ochoa JE, Sanchez A, Ruiz I. Epidemiología de la leptospirosis en una zona andina de producción pecuaria. Rev Panam Salud Publica (Revista on-line). 2000; 7 (5): 325-331.

8- Zuluaga León AG. Factores de riesgo asociados a leptospirosis en hatos bovinos de Pereira, 2002-2005. Investig. andina [Revista on-line]. 2009; 11 (9): 109-117.

9- Galton MM, Powers DK, Hall AM and Cornell RG. A rapid microscopic-slide screening test for the serodiagnosis of leptospirosis. Am. J. Vet. Res. 1958; 19: 505-12.

10- Oliveira AAF, Mota RA, Pereira GC, Langoni H, Souza MI Navegantes WA, Sá MEP. Seroprevalence of bovine leptospirosis in Garanhuns municipal district, Pernambuco State, Brazil. Onderstepoort. Journal of Veterinary Research. 2001; (68): 275279.
11- Luna MA, Moles LP, Gavaldón D, Nava C, Salazar F. Estudio retrospectivo de seroprevalencia de leptospirosis bovina en México considerando las regiones ecológicas. Rev. Cubana Med. Trop. [Revista on-line]. 2005; 57 (1): 28-31.

12- Pimienta CLRM, Castro V, Clementino IJ, Alves CJ, Fernandes LG, Brasil AWL, Santos CSAB, Azevedo SS. Leptospirose bovina no Estado da Paraíba: prevalência e fatores de risco associados à ocorrência de propriedades positivas. Pesq. Vet. Bras. (Revista on-line). 2014; 34 (4): 332-336.

13- Moles CLP, Gavaldón D, Torres BJ, Cisneros PMA, Aguirre SJ, Rojas SN. Seroprevalencia simultánea de leptospirosis y tres enfermedades de importancia reproductiva en bovinos del altiplano central de la República Mexicana. Rev. Salud Anim. 2002; 24 (2): 106-110.

14- Nicolino RR, Lopes LB, Rodrigues RO, Teixeira JFB, Haddad JPA. Prevalence and spatial analysis of antileptospiral agglutinins in dairy cattle - Microregion of Sete Lagoas, Minas Gerais, 2009/2010. Arq. Bras. Med. Vet. Zootec. (Revista on-line). 2014; $66(3): 648-654$.

15- Moles CLP, Cisneros MA, Gavaldón D, Rojas N, Torres JI. Estudio serológico de leptospirosis bovina en México. Rev Cubana Med. Trop. (Revista on-line). 2002; 54 (1): 24-27.

16- Motta JL, Clavijo JA, Waltero I, Abeledo MA. Prevalencia de anticuerpos a Brucella abortus, Leptospira sp. y Neospora caninum en hatos bovinos y bubalinos en el Departamento de Caquetá, Colombia. Rev. Salud Anim. (Revista on-line). 2014; 36 (2): 80-89.

17- Kocabiyik AL, Cetin C. Bovine leptospirosis in south Marmara region of Turkey: a serological survey. Revue Méd. Vét. 2004; 155 (12): 606-608. 